\title{
Mitigation of False Brinelling in a Roller Bearing: A Case Study of Four Types of Greases
}

\author{
Chih-Ling Lin ${ }^{1} \mathbb{D} \cdot$ Khosro Fallahnezhad $^{2} \cdot$ Osama Brinji $^{1,3} \cdot$ Paul A. Meehan ${ }^{1}$
}

Received: 1 November 2021 / Accepted: 23 December 2021 / Published online: 28 January 2022

(c) The Author(s) 2022

\begin{abstract}
Four commercial greases with various thickeners and base oils were experimentally examined to compare their false brinelling wear resistance in a test rig simulating roller bearings during rail/sea transportation for the first time. Greases containing zinc dialkyl dithiophosphates (ZDDP) showed superior false brinelling reduction, evidenced by no visible wear mark in the raceways. The mechanism for false brinelling mitigation was shown to be from a ZDDP-induced tribofilm which decreases the friction and wear coefficient in the contact area. Surface chemical analysis showed that for grease lubricated fretting contacts, ZDDP-derived tribofilms can be generated in the presence of micro-sliding motions and energy dissipation at the contact interface at low frequency (i.e., 4-8 Hz), due to the mechanochemical reactions. For greases without ZDDP, false brinelling wear was reduced by $97 \%$ when using grease with a more abundant and less viscous oil, which bleeds readily from an open structured thickener. The results highlight the ability of ZDDP as an additive in grease to better protect roller bearings against false brinelling during rail/sea transportation.
\end{abstract}

Keywords False brinelling $\cdot$ Grease lubricant $\cdot$ Roller bearing damage $\cdot$ ZDDP

\section{Introduction}

False brinelling, a form of fretting wear, mainly occurs when a lubricated rolling bearing is stationary during transport either by sea or rail $[1,2]$. The identification of false brinelling is by the elliptical wear marks on the raceways in the axial direction at each roller position [3]. The false brinelling marks are caused by micromovements due to very low-amplitude oscillation/ vibration between the rolling elements and the raceways. If the false brinelling wear is left unaddressed, service failure of the bearing will arise due to excessive running vibration, production of oxide particles and subsequent fatigue damage to the bearing surface during service [4-6]. The definition of false brinelling and fretting

Chih-Ling Lin

jenny.lin@uq.edu.au

1 School of Mechanical and Mining Engineering, The University of Queensland, St Lucia, QLD 4072, Australia

2 College of Science and Engineering, Medical Device Research Institute, Flinders University, Tonsley, SA 5042, Australia

3 Department of Mechanical Engineering, Taibah University, Al-Medina, Kingdom of Saudi Arabia corrosion in the literature is inconsistent $[7,8]$ and it can be a challenge to distinguish between these two terms because they can occur simultaneously, depending on the operating conditions [9]. For example, false brinelling can escalate to fretting corrosion when the number of cyclic vibrations increases, resulting in a lubricant being squeezed out of the contact zones $[4,6,10,11]$. The above situation also leads to some challenges for a lubricant to replenish the contact areas, which was observed in roller bearings for wind turbine applications [11]. In the current paper, false brinelling is seen in an oil/grease lubricated rolling bearing subjected to small oscillations, whereas fretting corrosion is described in the unlubricated condition $[5,7,9,12]$.

Lubrication is one of the major methods to reduce false brinelling wear and prevent fretting damage [6]. Main contributions of the lubricant to reduce fretting wear are lowering coefficient of friction (COF) and inhibiting oxygen access to the fretting contact areas [13, 14]. For an oil lubricant, viscosity is considered one of most important parameters for preventing fretting wear [6]. The effect of oil viscosity on fretting wear reduction has been shown to be dependent on the amplitude of oscillatory motions [15-17]. An oil with lower viscosity is effective for decreasing fretting wear when the amplitude is less than $9 \mu \mathrm{m}$ [15]. When 
the amplitude is more than $60 \mu \mathrm{m}$, oil viscosity has less impact on fretting wear reduction $[15,16,18]$. The degree of amplitude $(A / D)$, defined by a ratio between amplitude under oscillatory motion $(A)$ and Hertzian contact diameter $(D)$, has been used to study the effect of oil viscosity in fretting behaviour $[11,16,17]$. The above studies found the fretting wear can be reduced using (1) an oil with greater viscosity when $A / D>1.5$ or (2) a less viscous oil when $A / D \leq 1$. Maruyama et al. [17] showed that fretting wear can also be reduced by increasing the oscillating velocity under the same degree of amplitude (i.e., $A / D=1.9$ ).

Grease, on the other hand, its mechanisms of fretting wear reduction are more complicated and different from oil due to the complexity of chemical formula $[6,14,17]$. Studies have focused the ability of wear reduction of oscillating ball and thrust bearings in relation to the base oil viscosity $[3,17]$, grease consistency [19] as well as types of thickeners $[3,14]$. Pittroff [19] reported that soft grease is less effective for fretting wear reduction due to its weak shear strength and adhesiveness. Contradicting results published by Yan et al. [3] and Kita and Yamamoto [20] showed soft grease is more effective due to the higher fluidity of the base oil. Recent work done by Maruyama et al. [17] highlighted the grease with high bleed oil does not contribute to fretting wear reduction when $A / D>1$; because the worked thickener entered the contact and formed a layer to reduce the fretting wear. In Saatchi's work [14], three types of greases were tested under rolling fretting (referred to as false brinelling) with the Fafnir test and sliding fretting with linear-oscillation (SRV) test. His results showed oil bleeding behaviour of different grease types influences false brinelling, which is not the case in sliding contact fretting. This work highlighted the bleed or oil release mechanisms of grease under rolling and sliding are essentially different, resulting from the direction of the forces, motion on the thickener particles and oil-thickener interaction.

The most studies on grease performance for fretting wear was performed by unidirectional tests via the Fafnir fretting test, SRV and impact fretting test $[3,14,17,19]$. However, from the authors' previous work [5], rolling bearings were found to experience rotational oscillating displacements and subjected to three dimensional vibrations during rail/ sea transportation. Hence, a false brinelling test rig was designed accordingly, allowing authors to investigate vibration amplitudes and different types of loadings on false brinelling damage in railway cylinder roller bearings [12]. The results showed that the radial load and the amplitude of rotational displacement are the main causes of false brinelling wear during rail/sea transportation [12]. Hence, to build on this previous discovery, and using the custom false brinelling test rig, the current work aims to study the effect of four different types of greases for mitigation of false brinelling of rolling bearings during rail/sea transport.
It is a widely believed that to prevent false brinelling, a grease with high bleed properties should be selected to provide the fretting contacts with oil, to prevent starvation. However, recent studies have shown, that for grease, oil bleed (and oil viscosity) only partially contribute to the false brinelling reduction $[14,17]$. Grease thickener, polyurea to be specific, is also effective for mitigation of false brinelling by forming a layer in the fretting contact areas [17]. Except for the above studies, there is little work on the effect of different greases on false brinelling reduction. In addition, for oil lubricated systems, antiwear (AW) and extreme pressure (EP) additives have been shown to protect the fretting contact if the oil bleed is insufficient. However, it is unclear if the same protective principal applies to grease lubricated systems.

To date, most research has studied AW additives in oil for the mitigation of false brinelling [8, 20-23], with very little research available on AW additive performance in grease for false brinelling mitigation. Zinc dialkyl dithiophosphates (ZDDP) is the most popular AW agent [24, 25 ] and has shown promising results in reducing fretting wear in oil lubricated conditions [8,21, 22]. Given that all research into AW additives in grease focuses on tribofilm formation mechanisms during operating service conditions [24, 26-28], this paper sets out to explore the possibility of ZDDP tribofilm formation under false brinelling conditions (i.e., non-service conditions).

This study provides the following contributions to the body of false brinelling knowledge:

- Experimentally determined effect of four types of greases (different base oils, thickeners and additives) on false brinelling reduction via a custom false brinelling test rig. This test rig simulated the false brinelling in cylinder roller bearings that occur during rail/sea transportation

- Evaluation of tribological properties of four greases by reciprocating wear tests, so the possible mechanisms for false brinelling reduction under different greases can be understood.

- Evaluate the possibility of using ZDDP as a functional AW additive to mitigate false brinelling wear under grease lubricated conditions.

The current work focuses on the investigation of four commercial greases (contains different thickener, oil and additives) in mitigating false brinelling occurrence during rail/sea transportation. The false brinelling resistance of tested greases was evaluated by the maximum wear depth measured on the raceways. To understand how different types of greases mitigate the false brinelling, reciprocating wear tests with pin-on-cylinder configuration were performed. COF values of each grease type was measured in terms of frequencies, normal pressures, and temperatures. 
Worn surfaces and the tested greases were examined via optical microscopy, SEM/EDS (scanning electron microscopy/energy-dispersive spectroscopy) and XPS (X-ray Photoelectron Spectroscopy) to establish the extent to which lubricant formulation can reduce false brinelling damage.

\section{Materials and Methodologies}

\subsection{Grease Lubricants}

Four commercially available greases were used to investigate their performance for mitigating false brinelling wear in a roller bearing. Table 1 summarizes the main properties of the greases. All greases have similar density between 0.88 and $0.9 \mathrm{~g} / \mathrm{cm}^{3}$. In this study, four types of new and unworked greases were used in the experiments, as this replicates the starting condition in which bearing assemblies would leave their place of manufacture. During the false brinelling, base oil can be released from the grease under the vibrations or small oscillations, that is to say, the grease is worked, as it would be during the transport of bearing assemblies by rail/ sea.

\subsection{False Brinelling Test Rig}

The custom false brinelling simulator test rig, illustrated in Fig. 1a, was designed and fabricated for the authors' previous work [12]. In the current work, the test rig was configured such that the tested cylinder roller bearing experienced lateral vibration while radial load was applied (Fig. 1b). These testing parameters are the main causes of false brinelling wear during sea and rail transportation found in authors' work [12]. The shaft was press fit into the inner race of a FAG NU1018 cylindrical roller bearing prior to the test. To investigate the impact of grease on mitigating false brinelling damage, cylinder roller bearings were tested under dry and grease lubricated conditions. Figure 1a illustrates the dry test was performed solely. For the grease lubricated conditions, as shown in Fig. 1a, a fresh and unused grease was applied on to the raceway. It is noted that two grease lubricated bearings, placed $90 \mathrm{~mm}$ apart, were able to perform the tests at the same time.

The allocation of test rig experiments is summarized in Table 2. All tested greases were worked for 8 days and at the frequency of $24 \mathrm{~Hz}$. These parameters were chosen based on field data, recorded during rail/sea transportation for roller bearings in the authors' other work [5, 12].

To further understand how different greases mitigate false brinelling damage, pin-on-cylinder reciprocating wear tests were performed, which enabled us to conduct investigations under more controlled conditions. That is to say, the reciprocating wear test is representative of a single contact between a rolling element and the raceway in a roller bearing; allowing friction and wear coefficient of different grease lubricated contacts to be measured, which can be challenging when using full bearing assemblies in the false brinelling test rig.

\subsection{Evaluation for False Brinelling Wear}

After completion of false brinelling test rig experiments, the inner races were rinsed to remove grease samples. Surface topography of the wear mark produced by dry and lubricated conditions were obtained with Taylor Hobson Talysurf i5 surface profiler. The evaluation of false brinelling wear under dry and grease lubricated conditions was achieved by measuring the maximum wear depth from the cross-section of each wear mark profile. For each testing condition, three scans were performed cross the wear mark to identify the maximum wear depth.

\subsection{Reciprocating Wear Tests}

The experiments were carried out using a tribometer (UMT, Bruker, USA). The tribometer was adapted to be a pin-oncylinder set-up as shown in Fig. 2. In the tests, a ping with a curved surface of $39.38^{\circ}$ was subjected to a normal load

Table 1 Characteristic of four commercial greases used in the study

\begin{tabular}{lllll}
\hline Grease & Li/M & PU/PFPE +E & Li/Syn & LiC/M+Syn \\
\hline Base oil & Mineral & PFPE and Ester & Synthetic & Mineral and synthetic \\
Thickener & Li 12-hydroxysterate & PU & Li hydroxysterate & Li-complex \\
NLGI grade & 3 & 2 & 2 & 1 \\
Consistency & Semi-solid & Buttery & Buttery & Soft \\
Viscosity at $40^{\circ} \mathrm{C}(\mathrm{cSt})$ & $110-120$ & 130 & 100 & 130 \\
Oil separation by FTM-321 at $100{ }^{\circ} \mathrm{C}$ & 0.6 & $\leq 4$ & $\leq 4$ & Not available \\
Worked penetration, $25^{\circ} \mathrm{C}(0.1 \mathrm{~mm})$ & 230 & 270 & $265-295$ & $310-340$ \\
Additives & EP & ZDDP & Not available & ZDDP \\
\hline
\end{tabular}

PFPE perfluoropolyether, $P U$ polyuria, $L i$ lithium 


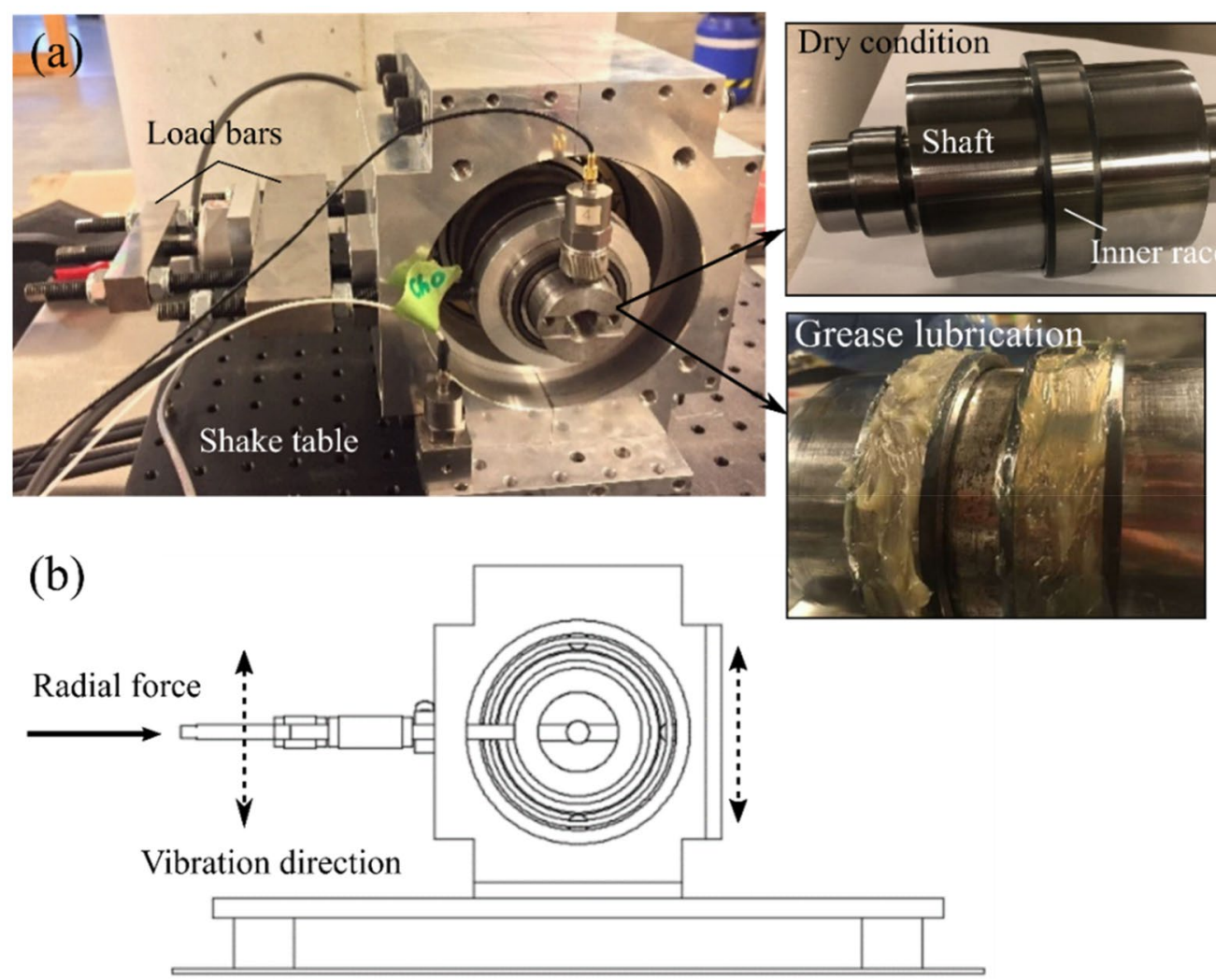

Fig. 1 a The configuration of custom false brinelling test rig and $\mathbf{b}$ schematic view of the lateral vibrational set-up used in the study. The images are adapted from authors' previous work [12]

Table 2 Testing conditions and parameters for the false brinelling test rig

\begin{tabular}{lllll}
\hline Test number & Bearing condition & $\begin{array}{l}\text { Fre- } \\
\text { quency } \\
(\mathrm{Hz})\end{array}$ & Static load (kg) & $\begin{array}{l}\text { Dura- } \\
\text { tion } \\
\text { (day) }\end{array}$ \\
\hline 1 & Dry & 24 & 80 & 8 \\
2 & Li/M & 24 & 160 & 8 \\
3 & PU/PFPE+E & & & \\
& Li/Syn & 24 & 160 & 8 \\
& LiC/Syn +M & & & \\
\hline
\end{tabular}

while reciprocating against the flat surface of a stationary cylinder. A small amount of fresh and unused grease was applied onto the estimated contact region of the friction pairs prior to the test. The tested cylinders and the pins were made of 52,100 high carbon bearing quality steel with the hardness is similar to the railway bearings [5, 12]. The diameter and width of the cylinder is $34.989 \mathrm{~mm}$ and $8.730 \mathrm{~mm}$, respectively. The part of the pin in contact with the bearing had a diameter of $4 \mathrm{~mm}$.

Table 3 specifies the experimental conditions for each type of grease, and each test was repeated two times. The amplitude of the sliding is $250 \mu \mathrm{m}$. Each test was conducted

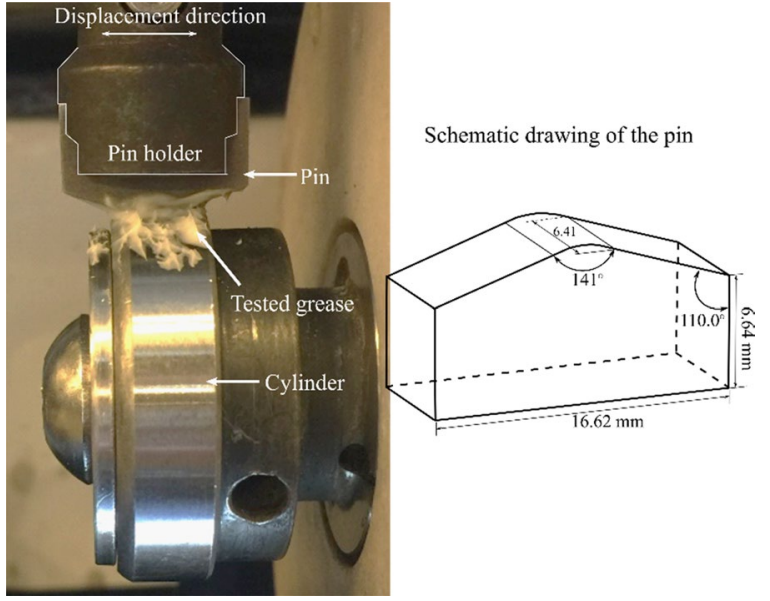

Fig. 2 Pin-on-cylinder configuration tribometer used for reciprocating wear tests. Noted the pin is wider than the cylinder width

for a fixed number of 200,000 cycles so that all experienced the same sliding distance. This means tests performed under low frequency lasted longer than those at the high frequency. Overall, greases were worked between 7 and $27 \mathrm{~h}$. The oscillating velocity is from 29.9 to $119.9 \mathrm{~mm} / \mathrm{s}$ depending on the frequencies. The applied normal loads were $4 \mathrm{~kg}$ and 
Table 3 Experimental parameters of the reciprocating wear tests

\begin{tabular}{lllrll}
\hline Grease & $\begin{array}{l}\text { Maximum contact } \\
\text { pressure }(\mathrm{MPa})\end{array}$ & $\begin{array}{l}\text { Hertzian contact } \\
\text { radius }(\mu \mathrm{m})\end{array}$ & $A / D$ & Frequency $(\mathrm{Hz})$ & Temperature $\left({ }^{\circ} \mathrm{C}\right)$ \\
\hline $\mathrm{Li} / \mathrm{M}$ & 340 & 11.5 & 10.87 & $2,4,6,8$ & 25 \\
& 700 & 20 & 6.25 & 4 & 25 \\
$\mathrm{PU} / \mathrm{PFPE}+\mathrm{E}$ & 340 & 11.5 & 10.87 & $2,4,6,8$ & 25 \\
& 700 & 20 & 6.25 & 4 & $25,45,85$ \\
$\mathrm{LiC} / \mathrm{Syn}+\mathrm{M}$ & 340 & 11.5 & 10.87 & $2,4,6,8$ & 25 \\
& 700 & 20 & 6.25 & 4 & $25,45,85$ \\
$\mathrm{Li} / \mathrm{Syn}$ & 340 & 11.5 & 10.87 & $2,4,6,8$ & 25 \\
& 700 & 20 & 6.25 & 4 & 25 \\
\hline
\end{tabular}

$10 \mathrm{~kg}$, equivalent to $340 \mathrm{MPa}$ and $700 \mathrm{MPa}$, respectively. Greases were tested under four different frequencies and two different pressures at ambient temperature. Two extra sets of reciprocating wear tests were performed on ZDDP-containing greases, PU/PFPE $+\mathrm{E}$ and $\mathrm{LiC} / \mathrm{Syn}+\mathrm{M}$ as Shown in Table 3. For each grease type, the tests were carried out with a normal pressure of $700 \mathrm{MPa}, 4 \mathrm{~Hz}$ and 200,000 cycles at temperatures of $45^{\circ} \mathrm{C}$ and $85^{\circ} \mathrm{C}$, respectively.

It should be noted that the vibration direction subjected to bearings in false brinelling test rig experiments is different from the sliding direction in the reciprocating wear tests, in addition, a higher frequency of $24 \mathrm{~Hz}$ was used in the false brinelling experiments. The parameters used in the false brinelling test rig were set accordingly to simulate false brinelling occurring during rail/sea transportation [5, 12]. However, due to the limitations of the tribometer, both the sliding direction and frequencies used in the reciprocating wear tests had be adjusted. Hence, the comparison between two tests cannot be made directly. As mentioned earlier, the reciprocating wear test was used as a supplementary test, which aimed to evaluate the tribological properties of four greases, under controlled laboratory conditions, so that the mitigation of false brinelling under different types of greases can be better understood.

\subsection{Evaluation of Tribological Properties of Greases}

During the tests, the vertical and lateral forces were recorded by the instrument, allowing for the COF to be determined. After completion of each reciprocating wear test, the mean $\mathrm{COF}$ of each grease type was calculated when the COF value reached a steady state (between 50,000 and 150,000 cycles). Tested grease samples were collected and tested cylinders were rinsed with n-hexane to remove supernatant grease. Wear marks on the cylinders were located by optical microscopy. SEM/EDS (JEOL 7001, JEOL, Japan) was used to examine the (1) topography of the worn surfaces and
(2) the composition of the worn surfaces produced through the four greases. For PU/PFPE + E and LiC/Syn + M, XPS analysis was carried out on the worn surface if the chemical compositions associated with ZDDP-induced tribofilm were identified by SEM/EDS. Therefore, possible microscopic mechanisms of the formation of the tribofilm under low oscillations can be confirmed. A XPS survey spectrum was acquired first, followed by a narrow scan to determine the relative atomic compositions of tribofilms. The charge of the XPS specimen was corrected by carbon binding energy to $284.8 \mathrm{eV}$. The microstructure of each grease type was imaged using SEM before/after the reciprocating tests. This is to examine potential structural change in thickener in relation to the small oscillations. The sample preparation of grease samples and set-up for SEM examination can be found in the authors' other work [26].

\subsection{Oil Bleed}

The ability of oil to be released, or bleed, from grease and enter the contact is important for rolling bearing applications [27]. The base oil released from thickener under fretting wear process can be different from the oil bleed from the static grease (indicated as oil separation by grease manufactures) [19]. This is because the oil separation is measured by putting grease in a cone sieve and the relative amounts of oil which are bled out due to gravity, as opposite to bleeding out under small-amplitude oscillation for false brinelling. Hence, oil bleed during false brinelling is considered as 'dynamic bleed' in this study. The 'dynamic bleed' was measured and characterized by centrifuge method [26, 28-30]. Approximately $0.5 \mathrm{~g}$ of grease was placed in a filter centrifuge tube, the refrigerated centrifuge was then spun at centrifugal force of $10,000 \times g$ at a constant temperature of $35^{\circ} \mathrm{C}$. The base oil recovered from the grease was weighed every $5 \mathrm{~h}$. The weight fraction of base oil was then estimated by weight of the bled oil divided by that of the sampled grease. 


\section{Results}

In the following section the comparison in false brinelling wear between dry and lubricated conditions (four grease types) are presented. The tribological properties of four greases (i.e., $\mathrm{COF}$ ) are evaluated by reciprocating wear tests. The worn surfaces are analysed morphologically and chemically. The microstructure of tested grease and the oil bleed are presented.

\subsection{False Brinelling Wear in Dry and Grease Lubricated Conditions}

False brinelling test rig experiments were performed under dry and grease lubrication conditions. The wear marks on the raceway were inspected and their maximum wear depths were estimated for assessment of false brinelling wear. Figure 3 shows the representative surface topography of observed wear marks, their corresponding cross-sections as well as the measured maximum wear depths. Overall, longer and wider wear marks were produced under unlubricated conditions (Fig. 3a). The average width of the wear marks decreased $80 \%$ ( $0.5 \mathrm{~mm}$ for dry and $\sim 0.1 \mathrm{~mm}$ for grease conditions) in lubricated conditions. It is clear from Fig. $3 \mathrm{~b}$ that the maximum depth of wear profiles reduced significantly by $~ 92 \%$ in $\mathrm{Li} / \mathrm{M}$ and $97 \%$ in $\mathrm{Li} / \mathrm{Syn}$ compared to the dry condition. No wear mark was observed when the tests were performed using PU/PFPE $+\mathrm{E}$ and $\mathrm{LiC} / \mathrm{Syn}+\mathrm{M}$.

\subsection{Evaluation of Tribological Properties of Tested Greases}

To understand the mitigation of false brinelling under four different greases found in the false brinelling test rig experiments, reciprocating wear tests were performed. A series of different reciprocation frequencies and two normal pressures were applied with all grease lubricated contacts.

\subsubsection{COF Measurement}

Figure 4a shows the variations of COF values of four greases for given testing cycles. Evidently, $\mathrm{LiC} / \mathrm{Syn}+\mathrm{M}$ shows an overall $64 \%$ lower COF value than LiC/Syn, PU/PFPE $+\mathrm{E}$ and $\mathrm{Li} / \mathrm{M}(\mathrm{COF}=0.1-0.11)$. Under the same testing condition, $\mathrm{COF}$ values of PU/PFPE $+\mathrm{E}$ and $\mathrm{Li} / \mathrm{Syn}$ are identical throughout the wear tests.

Figure $4 \mathrm{~b}$ shows the mean COF values of tested greases under four frequencies ranged from 2 to $8 \mathrm{~Hz}$. The mean $\mathrm{COF}$ of each grease type was calculated when the $\mathrm{COF}$ value reached a steady state (between 50,000 and 150,000 cycles) as shown in Fig. 4a. LiC/Syn $+\mathrm{M}$ has the lowest $\mathrm{COF}$ values (below 0.05) than the other three types of greases under all test frequencies (Fig. 4b). Each grease type showed insignificant difference in $\mathrm{COF}$ with increasing frequency from 2 to $6 \mathrm{~Hz}$. However, COF values of $\mathrm{Li} / \mathrm{M}, \mathrm{Li} / \mathrm{Syn}$ and $\mathrm{LiC} / \mathrm{Syn}+\mathrm{M}$ increased about 23-30\% when the frequency increased from 6 to $8 \mathrm{~Hz}$. The result also shows that PU/ $\mathrm{PFPE}+\mathrm{E}$ is much less sensitive to the test frequencies with its COF values fluctuating slightly around 0.1 .

Figure $4 \mathrm{c}$ shows average COF values decrease as the normal pressure increases. The trend was observed in all grease types. The mean COF value decreased $30 \%$ for $\mathrm{Li} / \mathrm{M}, \sim 14 \%$ for PU/PFPE $+\mathrm{E}$ and $\mathrm{LiC} / \mathrm{Syn}+\mathrm{M}$ and only $5 \%$ for $\mathrm{Li} / \mathrm{Syn}$. For ZDDP-containing greases, tests were carried out at two higher temperatures. The results, from Fig. 4d, show PU/ $\mathrm{PFPE}+\mathrm{E}$ and $\mathrm{LiC} / \mathrm{Syn}+\mathrm{M}$ performed similarly well at all three temperatures tests. LiC/Syn $+\mathrm{M}$ outperformed the PU/ $\mathrm{PFPE}+\mathrm{E}$ by $66.7 \%$ reduction in $\mathrm{COF}$ values.

\subsubsection{Topography of Worn Surfaces}

Figure 5 shows the optical microscopy images of wear marks on the cylinders after the reciprocating wear tests as well as the SEM micrographs of worn surface located in the centre of each wear mark. It is clear that the introduction of $\mathrm{LiC} /$ Syn $+M$ resulted in a decrease in wear mark diameter and the wear mark is less evident (Fig. 5d). Hence, the wear produced using LiC/Syn $+\mathrm{M}$ was not as severe as in other three types of greases. SEM micrographs also revealed that $\mathrm{LiC} / \mathrm{Syn}+\mathrm{M}$ leads to a relatively smooth wear surface with fewer grooves present on the worn surface compared to the other three types of greases. The results reflect to the $\mathrm{COF}$ measurement seen in Fig. 4, where LiC/Syn $+\mathrm{M}$ has the lowest COF $(<0.05)$ among all tested greases.

The typical morphology of worn surfaces in $\mathrm{Li} / \mathrm{M}$ (Fig. 5a), PU/PFPE + E (Fig. 5b) and Li/Syn (Fig. 5c) were characterized by deep and shallow grooves. Other features, such as pitting and adhesive wear, also appear at the contact worn surfaces for three grease types. It is inconclusive if the shallow/deep groove features seen on the worn surfaces were the products of the reciprocating wear tests, rather the initial machining trace surface topography of the cylinders. Nevertheless, relatively smoother surfaces observed in $\mathrm{LiC} /$ Syn $+M$ might be an additional contributing factor for the reduction in wear damage in false brinelling test rig tests.

\subsubsection{Chemical Compositions of Worn Surfaces}

EDS analysis was performed on the same worn surface shown in Fig. 5. It essentially gives an indication of the interaction of the tribological surface with the additives within different greases. Moreover, the analysis provides evidence for the formation of a protective layer on the worn 
(a)

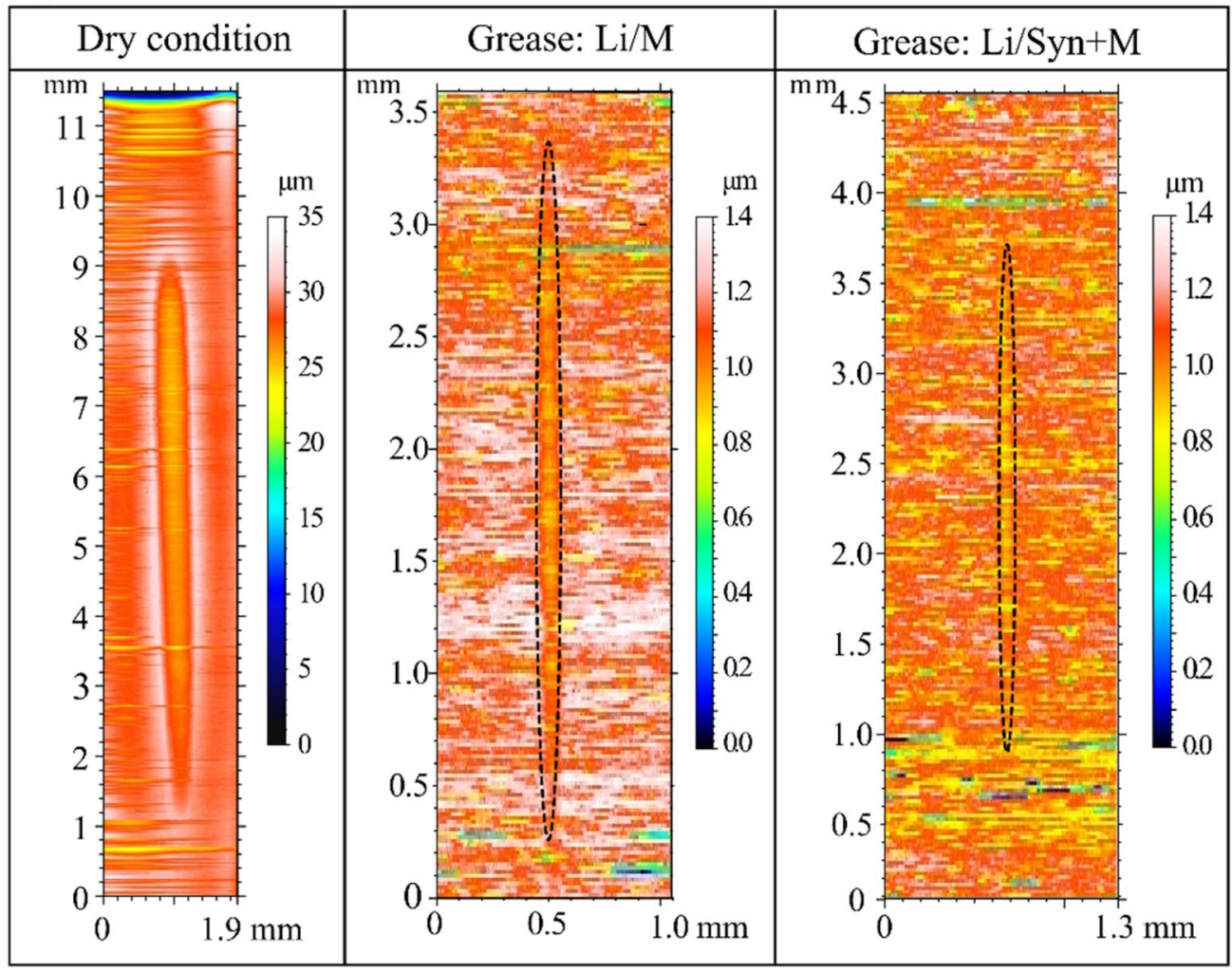

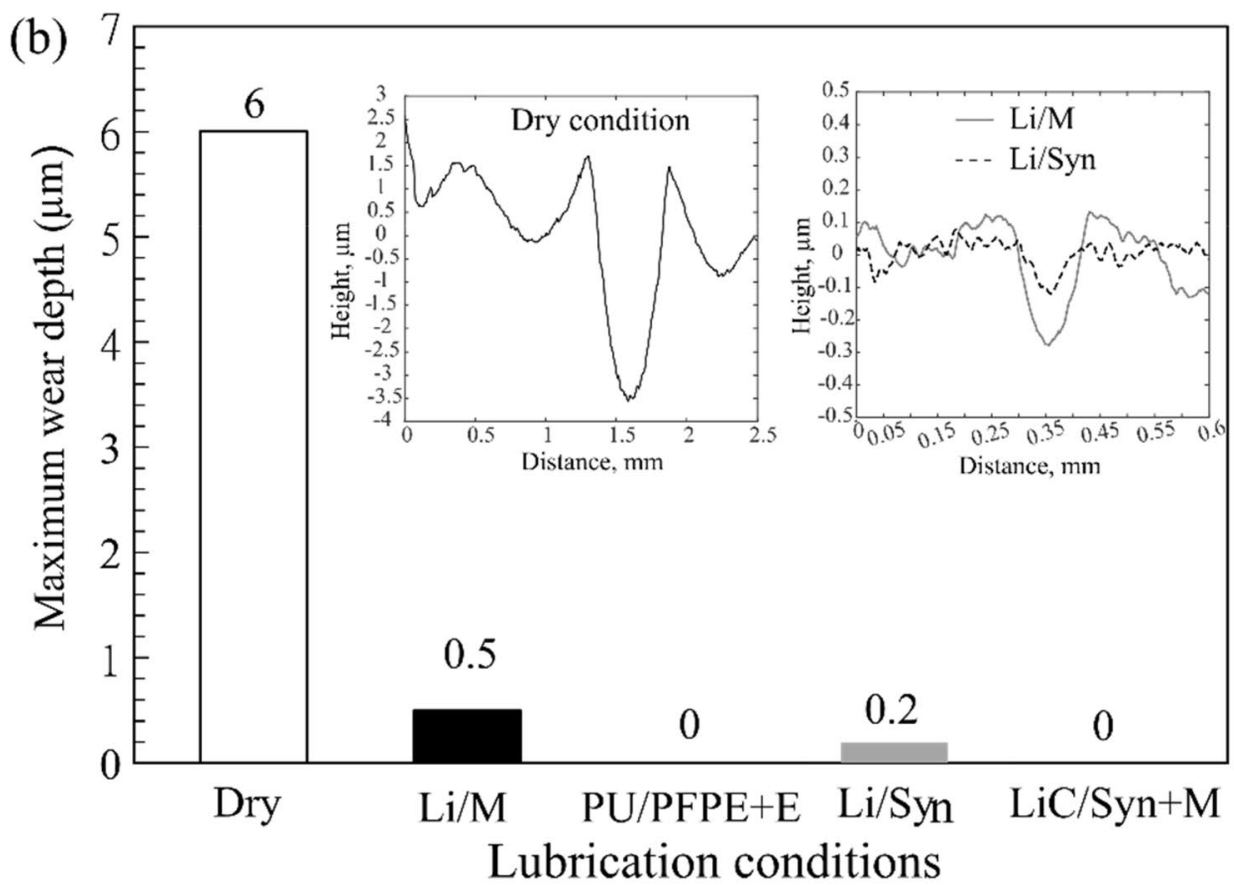

Fig. 3 Representative a 3D top view surface topography of wear marks) obtained under dry and lubrication conditions. b The comparisons of cross-section wear profiles and measured maximum wear depths for different testing conditions

surface and of its chemical composition. Figure 6a shows Fe (iron), $\mathrm{Si}$ (silicon) and $\mathrm{Cr}$ (chromium) peaks originate from the cylinder material. The lower concentration of $\mathrm{Zn}$ (zinc), $\mathrm{P}$ (phosphorous) and $\mathrm{S}$ (sulfur) were found within the ZDDP-containing greases (PU/PFPE + E, LiC/ Syn $+\mathrm{M})$ and EP-containing grease $(\mathrm{Li} / \mathrm{M})$. As such, EDS 

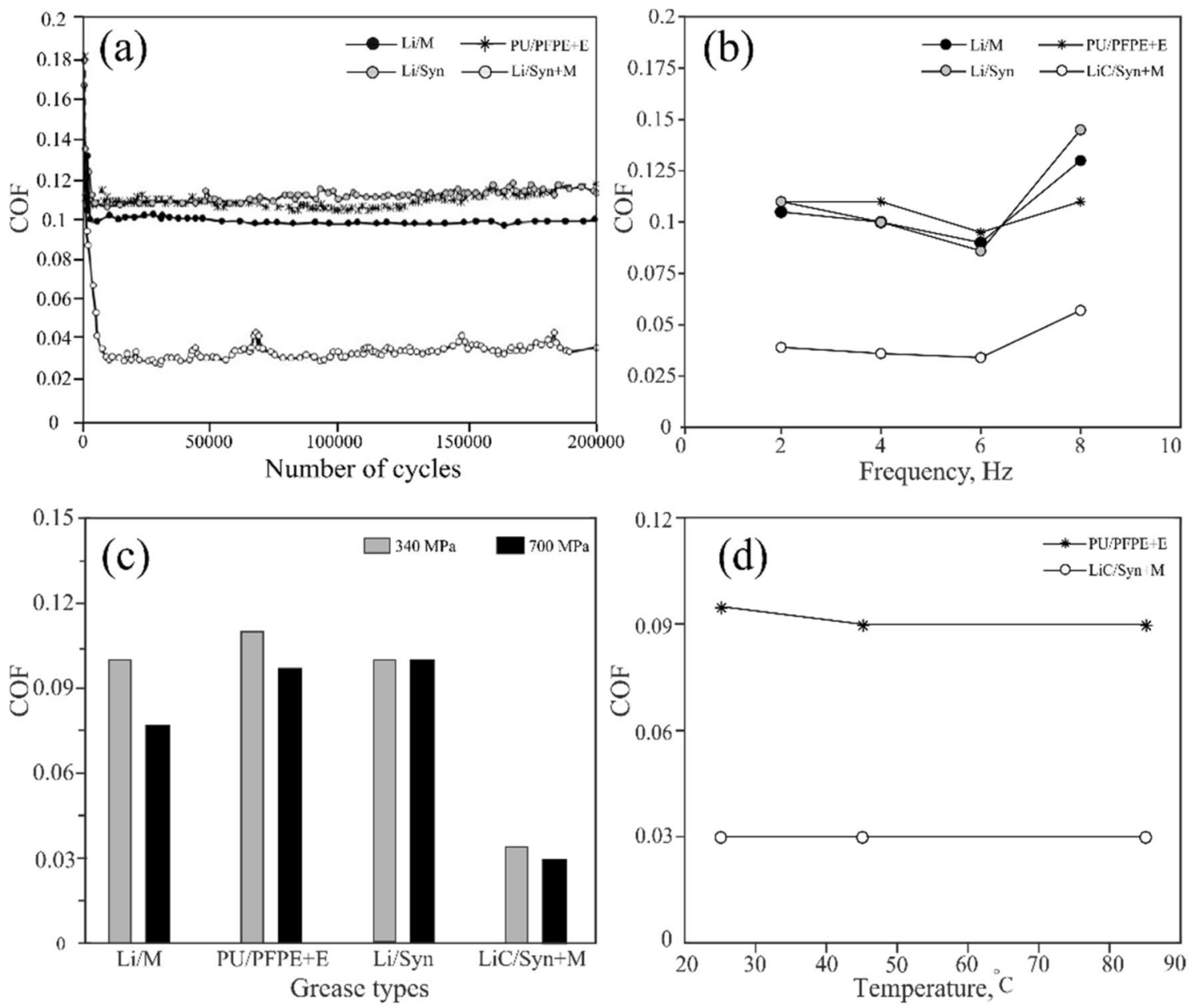

Fig. 4 a Variations of COF for four greases at $4 \mathrm{~Hz}, 340 \mathrm{MPa}$ and ambient temperature. $\mathbf{b}$ Mean COF values using four types of greases at 2, 4, 6 and $8 \mathrm{~Hz}$. c Mean COF values using four types of greases

result suggested, for $\mathrm{Li} / \mathrm{M}$ grease, sulfur in the EP reacted with the contact surface forming a sulfide layer during the reciprocating wear tests. The result indicates the interaction of the worn surface with the additives within the greases. Particularly within ZDDP-containing greases (Fig. 6b, c), the presence of $\mathrm{Zn}, \mathrm{P}$ and $\mathrm{S}$ indicate the possibility of a protective tribofilm on the worn surface, which was confirmed using XPS. Figure $6 \mathrm{~b}$ and $\mathrm{c}$ demonstrate that the intensities of ZDDP-related peaks vary depending on the selected areas in the worn surfaces. EDS results also indicate the ZDDPinduced tribofilm can either form at higher frequency and room temperature (Fig. 6a) or at a lower frequency with a combination of a higher normal pressure (Fig. 6b, c).

\subsubsection{XPS Analysis of Tribofilms}

To identify the chemical compounds of tribofilms and to support the findings from SEM/EDS analysis (shown in Fig. 6), a more surface sensitive technique, XPS, was carried

at $4 \mathrm{~Hz}, 340$ and $700 \mathrm{MPa}$. d Mean COF values using two types of ZDDP-containing greases at 25,45 and $85^{\circ} \mathrm{C}$

out on the worn surfaces for the tests using PU/PFPE $+\mathrm{E}$ (Fig. 7) and LiC/Syn + M (Fig. 8). The survey spectra, shown in Fig. 7a and c, were obtained from PU/PFPE +E and $\mathrm{LiC} / \mathrm{Syn}+\mathrm{M}$, respectively. These spectra were used for peak identifications associated with ZDDP. For both greases, the most intense component in the $\mathrm{C} 1 \mathrm{~s}$ peaks at $284.8 \mathrm{eV}$ was referred to $\mathrm{C}-\mathrm{C}[31,32]$. Minor contributions found at $286.2 \mathrm{eV}$ were assigned to ester/alcohol groups (i.e., $\mathrm{C}-\mathrm{OH}$, $\mathrm{C}-\mathrm{O}-\mathrm{C})$. The peak located at $288.8 \mathrm{eV}$ is assigned to $\mathrm{C}=\mathrm{O}$ or metal carbonate $[32,33]$. For PU/PFPE $+\mathrm{E}, \mathrm{F}$ 1s spectra at $688.5 \mathrm{eV}$ indicates the present of metallic fluoride on the worn surface (Fig. 7a). The result indicates the reaction between the PFPE oil and the metal surface can be induced by the small oscillations.

The curve fitting was performed on the $\mathrm{Zn} 2 \mathrm{p}_{3 / 2}$ signal for PU/PFPE +E (Fig. 8a) and LiC/Syn + M (Fig. 8e), respectively. For both greases, results correspond to the metallic zinc and possibly metal sulphide $(\mathrm{ZnS})[34,35]$ due to the detection of S (Fig. 8d for PU/PFPE+E and Fig. 8h for LiC/ 
Fig. 5 Representative optical microscopic images (left) and SEM micrographs (right) of wear marks on the cylinders under a $\mathrm{Li} / \mathrm{M}, \mathbf{b}$ PU/PFPE + E, c Li/Syn and $\mathbf{d} \mathrm{LiC} / \mathrm{Syn}+\mathrm{M}$ at $4 \mathrm{~Hz}, 340 \mathrm{MPa}$ and ambient temperature
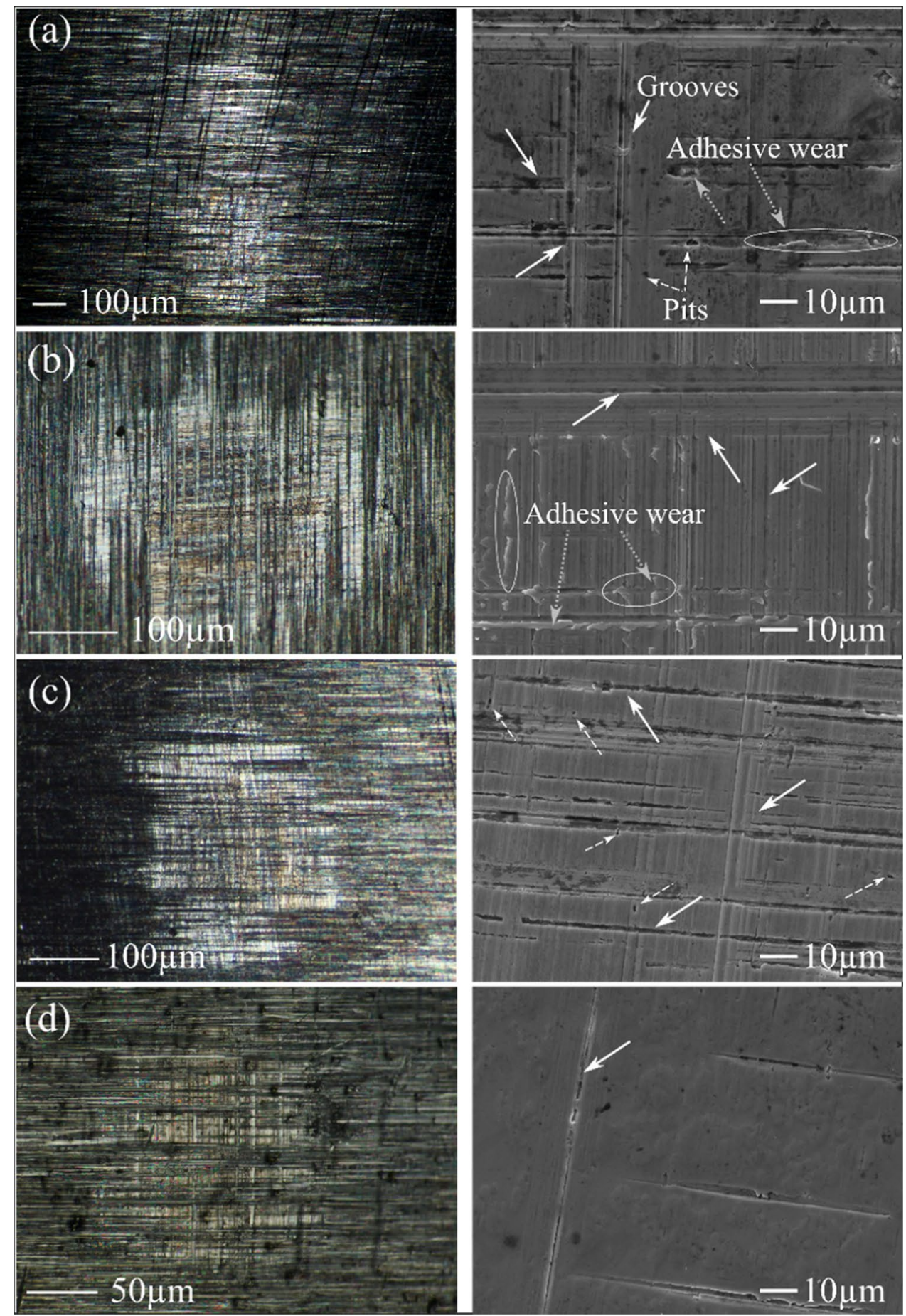

Syn $+M)$. The lower energy peak in the deconvoluted $\mathrm{Zn}$ spectrum for PU/PFPE $+\mathrm{E}$ is attributed to the oxide form of Zn (Fig. 8a). The iron spectra (PU/PFPE in Fig. 8b and LiC/Syn $+\mathrm{M}$ in Fig. 8f) consists of two main peaks with $\mathrm{Fe} 2 \mathrm{p}_{3 / 2}$ at $710.81 \mathrm{eV}$ and $\mathrm{Fe} 2 \mathrm{p}_{1 / 2}$ at $\sim 724.58 \mathrm{eV}$. The lower binding energy of $\sim 707.20 \mathrm{eV}$ is assigned to iron disulfide $\left(\mathrm{FeS}_{2}\right)$ [36-38]. The main component at $\sim 710.3 \mathrm{eV}$ is the peak from iron oxide (Ш) [31, 34, 35]. FeS is also identified at the higher binding energy of $\sim 712 \mathrm{eV}$.
$\mathrm{O} 1 \mathrm{~s}$ spectrums for $\mathrm{PU} / \mathrm{PFPE}+\mathrm{E}$ and $\mathrm{LiC} / \mathrm{Syn}+\mathrm{M}$ are shown in Fig. 8c and g, respectively. For both greases, the lower binding energy at $\sim 529 \mathrm{eV}$ are assigned to zinc and iron oxide, followed by the peak which is assigned to metal hydroxides at $\sim 531.3 \mathrm{eV}[31,34,38]$. Two peaks at higher binding energies ( 533-535 eV) are further separated into (1) non-Bridging-Oxygen (NBO) peak possibly originating from sulfate $\left(\mathrm{SO}_{4}\right), \mathrm{SiO}_{2}$ or hydroxides as well as (2) a Bridging-Oxygen (BO) peak linked to water [31, 


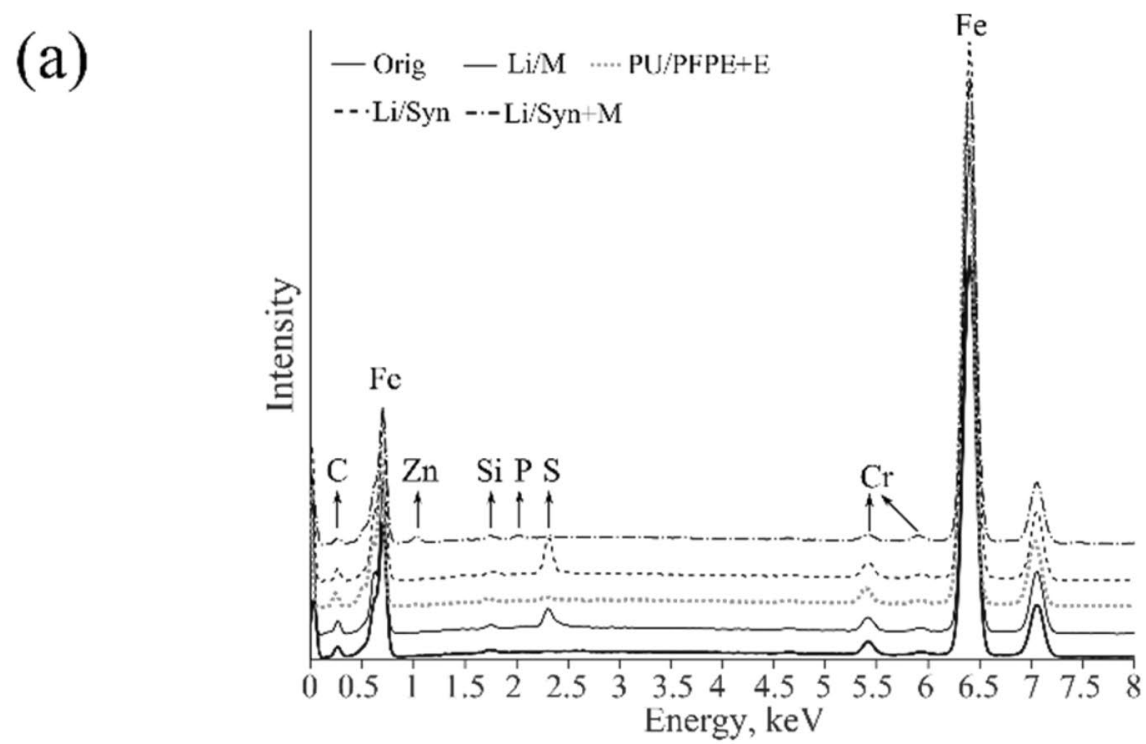

(b)

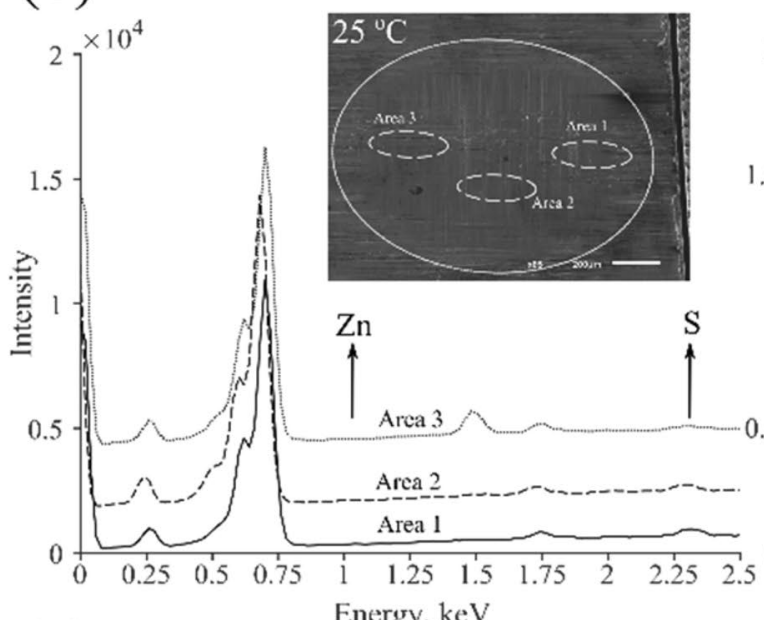

Grease: PU/PFPE+E

(c) Energy, keV
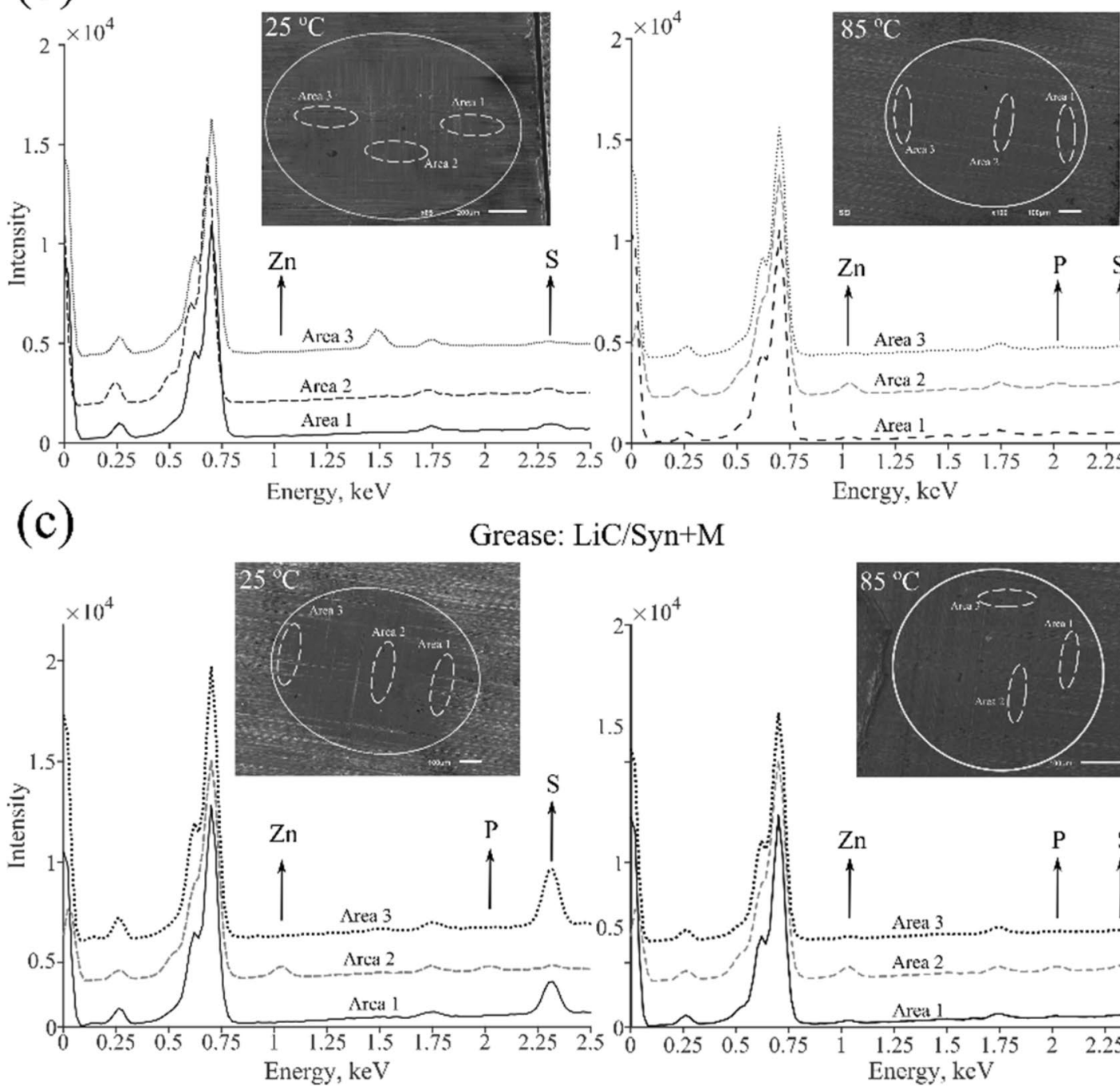

Grease: LiC/Syn+M

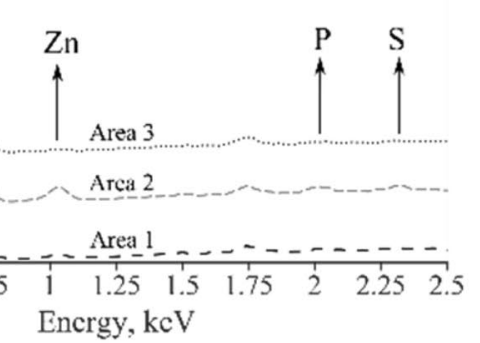


(a)

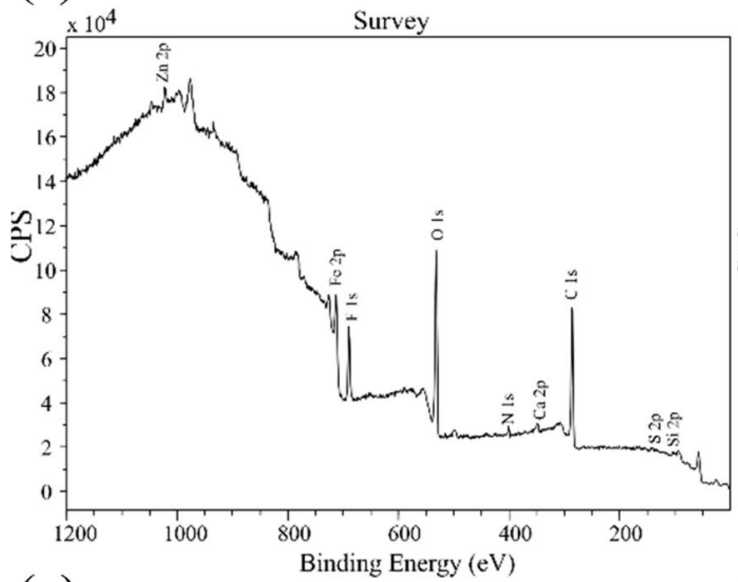

(c)

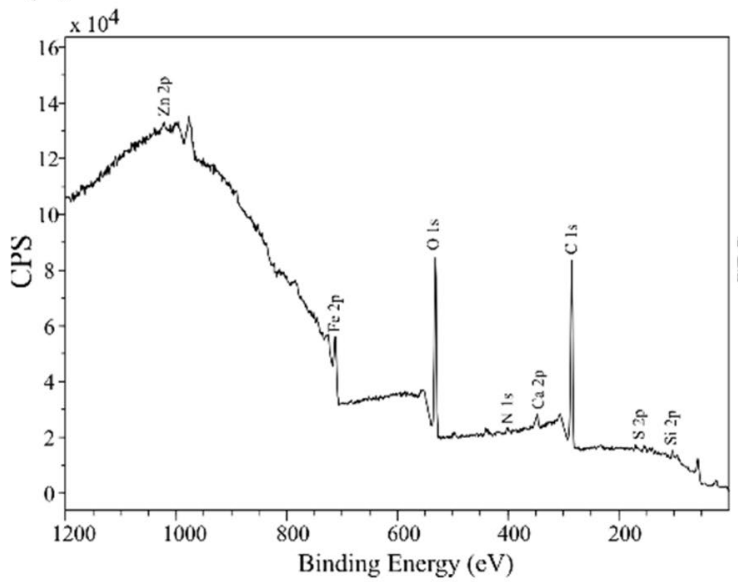

(b)

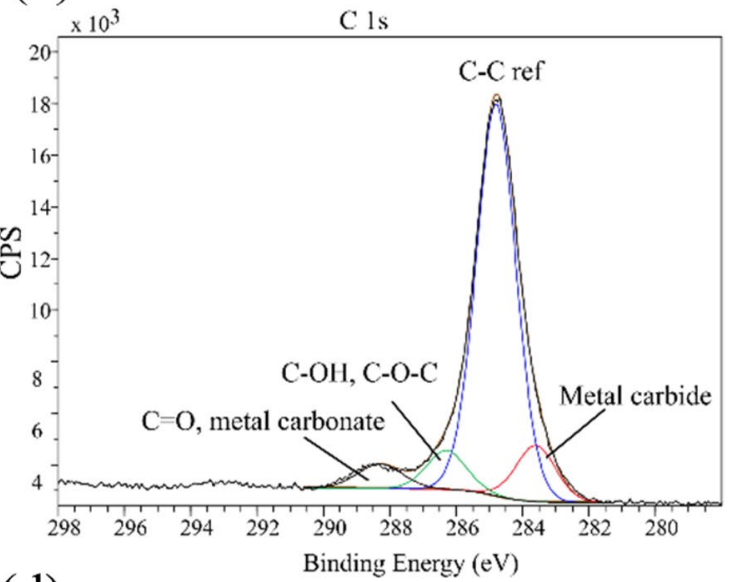

(d)

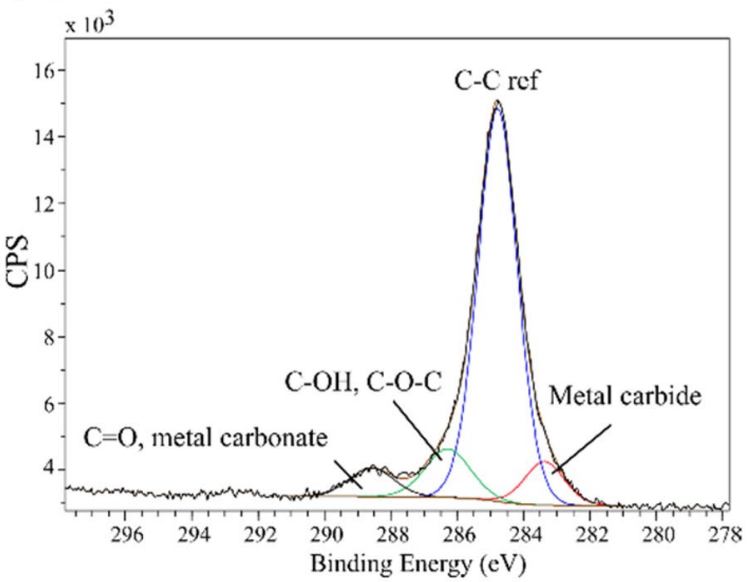

Fig. 7 XPS survey and high resolution spectra of $\mathrm{C} 1 \mathrm{~s}$ for PU/PFPE $+\mathrm{E}(\mathbf{a}, \mathbf{b})$ and $\mathrm{LiC} / \mathrm{Syn}+\mathrm{M}(\mathbf{c}, \mathbf{d})$, respectively. Test condition was $8 \mathrm{~Hz}$, $340 \mathrm{MPa}$ and ambient temperature

$32,36,37]$. In the case of $\mathrm{S} 2 \mathrm{p}$, one stronger peak was detected at $161.8 \mathrm{eV}$ and it was assigned to the sulfide group, where the weak peak at $167.9 \mathrm{eV}$ was assigned to the sulfate group [32, 35, 37].

\subsubsection{Microstructural Characterisation of Thickener}

Figure 9 shows the microstructure of different types of thickeners in four grease types taken before and after the reciprocating wear tests. The results show the $\mathrm{Li} / \mathrm{M}$ (Fig. 9a), Li/Syn (Fig. 9c) and LiC/Syn + M (Fig. 9d) greases show a fibrous microstructure. The average diameter of fibre is around $76-120 \mathrm{~nm}$. On the other hand, a completely different microstructure was exhibited by PU/PFPE + E grease (Fig. 9b), where PU presented in platelet and short fibrous shapes. The diameter of platelets is around $30-117 \mathrm{~nm}$, and the short fibers are around
45-87 nm. After the tests, the thickener of Li/M (Fig. 9a) became loosely bound compared to the initial stage. For $\mathrm{Li} / \mathrm{Syn}$ and $\mathrm{LiC} / \mathrm{Syn}+\mathrm{M}$, small morphological changes suggested that the fibrous thickener was broken sown slightly. For Li/M (Fig. 9a), the overall structural integrity did not appear compromised significantly after the tests.

\subsubsection{Oil Bleed}

The measurement of oil bleed in different greases offers an explanation of the false brinelling mitigation of the four different greases. The capacity of oil bleed from thickener was examined and the results shown in Fig. 10. The initial slope of each curve (first $5 \mathrm{~h}$ in Fig. 10) was used to measure the ease of base oil bleeding per grease sample. The result shows oil is easier to be released from the greases with fibrous thickener $(\mathrm{Li} / \mathrm{M}, \mathrm{Li} / \mathrm{Syn}$ and $\mathrm{LiC} / \mathrm{Syn}+\mathrm{M})$ than $\mathrm{PU}$ grease. 

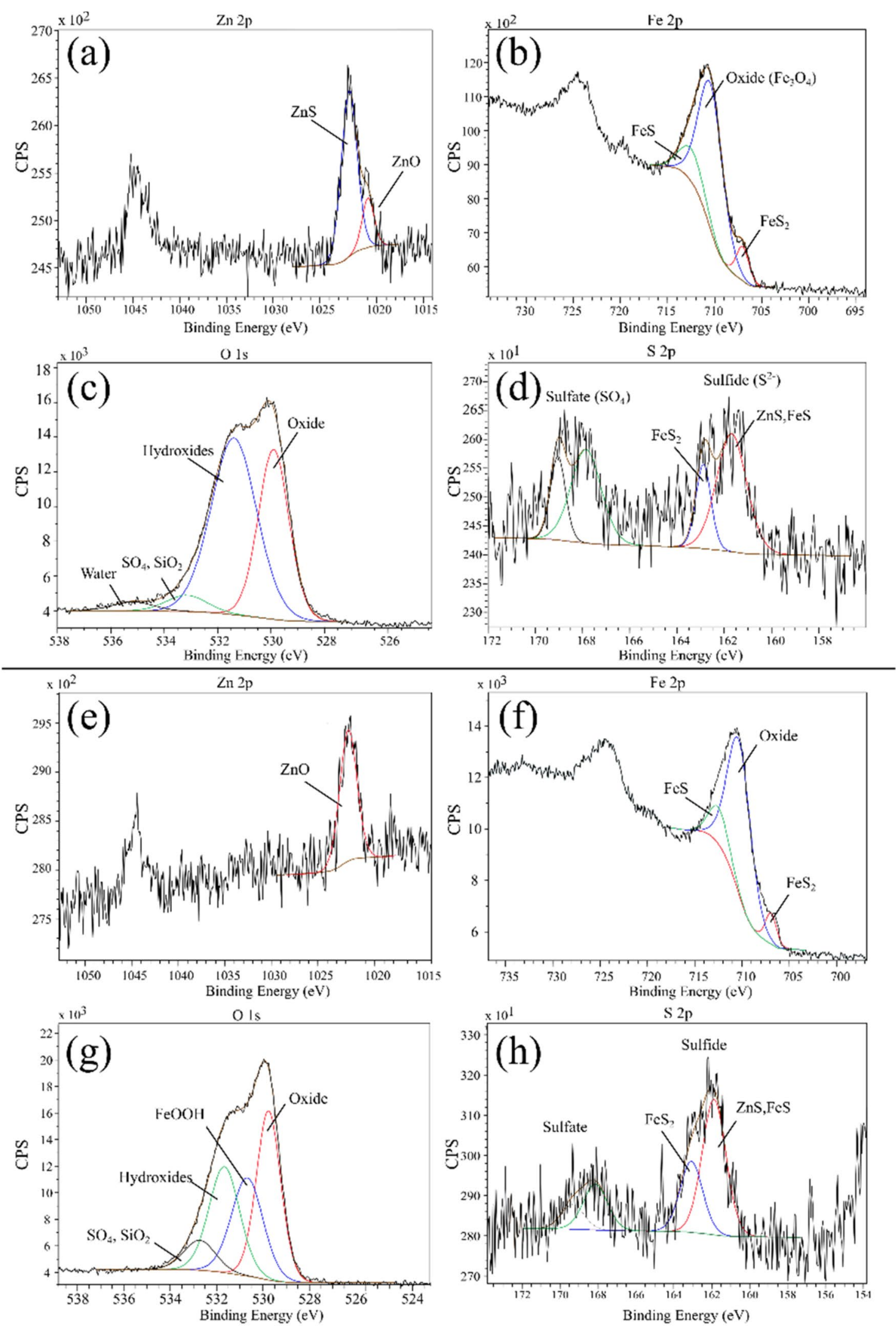

Fig. 8 XPS high resolution spectra of the worn surfaces under a-d PU/PFPE + E and e-h LiC/Syn + M (test condition, 8 Hz, $340 \mathrm{MPa}$, ambient temperature) 


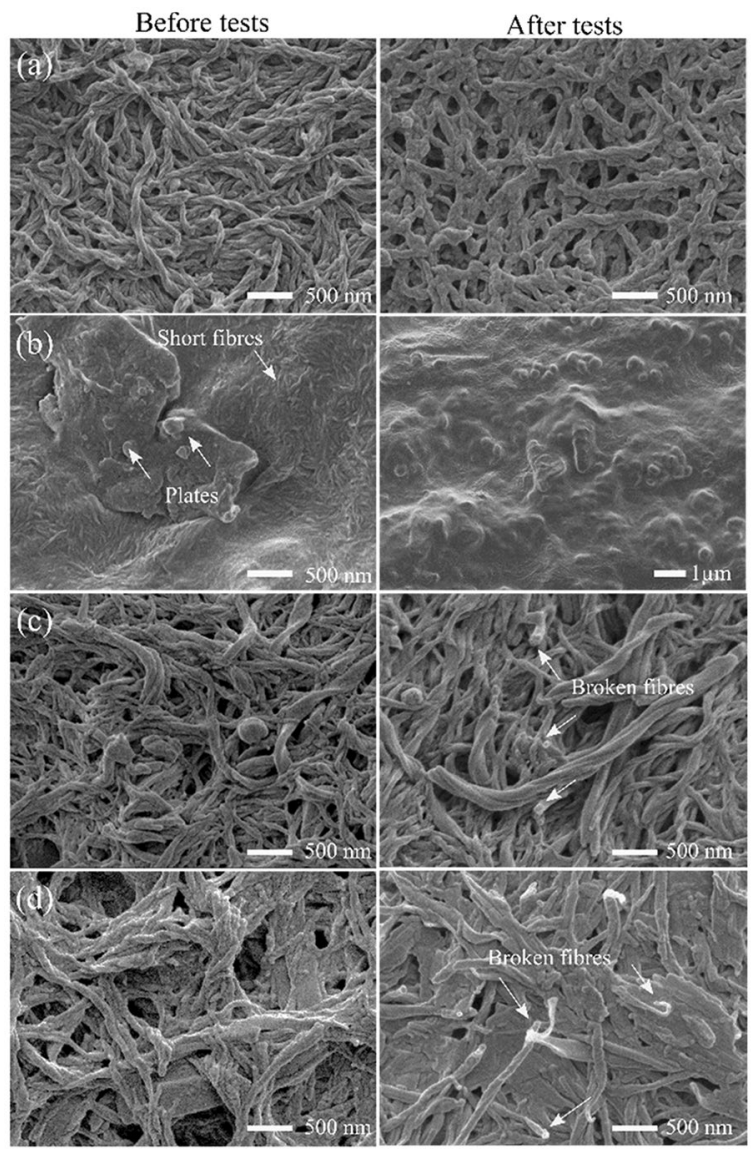

Fig. 9 SEM micrographs of microstructure of a Li/M, b PU/ $\mathrm{PFPE}+\mathrm{E}, \mathbf{c} \mathrm{Li} / \mathrm{Syn}$ and $\mathbf{d} \mathrm{LiC} / \mathrm{Syn}+\mathrm{M}$ taken before and after the reciprocating wear tests $\left(340 \mathrm{MPa}, 8 \mathrm{~Hz}, 25^{\circ} \mathrm{C}\right)$. Note the image a is adapted from authors' previous' work [26]

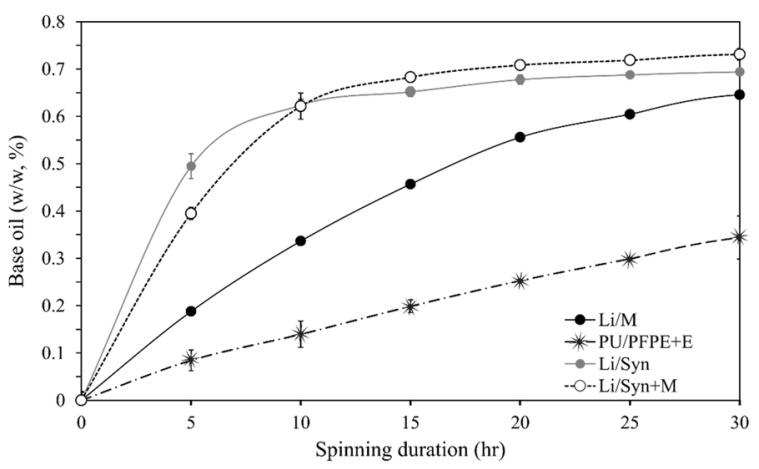

Fig. 10 Base oil bleeding rate of the tested grease samples

\section{Discussions}

As expected, all four types of greases reduced false brinelling wear compared to the dry condition. The results highlighted that the magnitude of false brinelling wear reduction is significantly influenced by the grease formula under the studied conditions. Differences in mechanisms for false brinelling reduction under different greases are compared and discussed. Two mechanisms could be explained the differences in the false brinelling mitigation results obtained from different types of greases. The mechanism for greases contain ZDDP are compared and discussed in Sect. 4.1 to Sect. 4.3. For $\mathrm{Li} / \mathrm{M}$ and $\mathrm{Li} / \mathrm{Syn}$, the mechanism for false brinelling reduction can be found in Sect. 4.4. This is followed the explanation for differences found between ZDDP in grease and EP in grease for false brinelling wear reduction.

\subsection{False Brinelling Wear in ZDDP-Containing Greases}

Two ZDDP-containing greases $(\mathrm{LiC} / \mathrm{Syn}+\mathrm{M}$ and $\mathrm{PU} /$ PFPF $+E$ ) were found to be superior for mitigating false brinelling wear. As shown in Fig. 3, no wear mark was visually observed on the raceways. The mechanism of mitigating false brinelling wear during sea/rail transport is due to the formation of a tribofilm by ZDDP on the fretting wear contacts. EDS and XPS analysis confirmed that a small oscillation frequency $(4-8 \mathrm{~Hz})$, low pressure $(<1 \mathrm{GPa})$ and ambient temperature can facilitate the ZDDP tribofilm growth on the worn surfaces. From Table 1 and Fig. 10, it is important to note that both greases contain higher viscous base oil compared to $\mathrm{Li} / \mathrm{M}$ and $\mathrm{Li} / \mathrm{Syn}$, and PU/PFPE $+\mathrm{E}$ demonstrated the worst 'dynamic bleed' among all tested greases (shown in Fig. 10). It can be inferred that oil bleed behaviour and oil viscosity do not, at least, directly influence the performance of false brinelling wear resistances in ZDDP-containing greases.

\subsection{Formation of Tribofilm}

In oil lubricated systems, it has been discovered that heat generated by sliding can stimulate the formation of ZDDP films [19, 25], but this has not previously been investigated in grease. Here, heat is referred to the localized and transient temperature rise on the rubbing surfaces and not due to the testing/environment conditions. Accordingly, heat can also be one of the drivers for promoting the tribofilm formation under grease lubricated conditions. Under the tested conditions in this study, it is likely the grease lubricated tests were operating under boundary lubrication regimes [39]. As a result, heat generated at asperity contact would occur and the resulting local shear stress and heat promoted ZDDP tribofilm formation.

It is well-document that ZDDP films can form with a combination of increased temperature (flash temperature) and shear of the lubrication film in oil lubricated system [8, 
$25,40]$. These factors are described as 'mechanochemical reactions', which drive the ZDDP tribofilm formation under the rubbing surface at low environmental temperatures $25^{\circ} \mathrm{C}$ to $85{ }^{\circ} \mathrm{C}$ [25]. Furthermore, in these oil lubricated systems, the ZDDP tribofilm forms even if there is no asperity contact due to EHL (Elastohydrodynamic Lubrication) regime, on the proviso that the shear stress is sufficiently high [40]. However, the shear of the lubrication film is unlikely to be the contributing factors for the ZDDP tribofilm formation in the current result. This is because the ZDDP-containing greases were tested at the lower range of parameters compared to other grease studies (i.e., frequency $>20 \mathrm{~Hz}$, pressure $>1 \mathrm{GPa}$ ) $[8,17,23]$. As a result, low frequencies (low sliding speeds, below $0.1 \mathrm{~m} / \mathrm{s}$ for $2-6 \mathrm{~Hz}$ ) and the shear stress within the contacts are considered very low (due to low contact pressure). The mechanical driver of tribofilm formation would have occurred as the grease was pushed out of the fretting contact regions during small sliding oscillatory movements; meaning that the contact is most likely operating under a boundary lubrication regime. As a result, asperity contact would occur and the resulting local shear stress and heat promoted ZDDP tribofilm formation.

As XPS analysis clearly shows that a layer of $\mathrm{Zn}, \mathrm{Fe}$ and $S$ were formed on the top of the worn surfaces. As such, the combination of a high intensity oxygen, with localised iron/zinc sulphide, iron-induced tribofilm has been seen in other studies [31, 34, 36, 41, 42]. Hence, the chemical driver of the ZDDP tribofilm formation can be explained by the mechanism proposed by Ito et al. [42], where the composition of the tribofilm can vary depending on the oxidation state of the initial contact area between the frictional pairs. That is to say, ZDDP molecules start to decompose in the event of sliding, resulting in a layer of zinc-rich, sulfur-free adsorption layer (i.e., $\mathrm{FeS}_{2}$ and $\mathrm{ZnS}$ ), where the initial tribofilm formation occurs on the iron oxide. The formation of polythio-phosphate may occur later in the reaction sequence once the temperature in the contact region rises, activating this reaction.

EDS results (Fig. 6b, c) showed the peak and intensity variations in tribofilm-related elements per selected areas. One explanation is that the thickness of the tribofilm in some selected areas are much less than the interaction volume of electron beam and the tested material $(\sim 2 \mu \mathrm{m})$. If that is the case, the relevant tribofilm peaks generated under tested conditions may be present but fall below the detection limit of the SEM/EDS techniques. Under the same principle, the quantitative comparison of the film composition between different grease conditions was not possible. Nevertheless, EDS analysis was able to confirm the growth of the tribofilm formation under the designated conditions. ZDDP-induced tribofilm under the studied conditions is rather patchy on the worn surfaces in this study. The present finding is different from the observations in oil lubrications, where ZDDP reaction layers grow quickly and easily on the rubbing surface, in as little time as $75 \mathrm{~min}$ and around $60 \mu \mathrm{m}$ thick [43, 44]. Additionally, a general observation of tribofilm formation under oil lubricants in the EHL regime occurs when a combination of temperature increase and shear of the lubricant takes place [8, 23, 25].

On the other hand, grease has more complicated lubrication regimes compared to oil due to the presence of thickener in grease. As a result, the fully flooded contact and the replenishment condition are not as straightforward as has been observed in oil [27, 45, 46]. When considering false brinelling, the grease lubricated bearings tend to operate in the starved EHL regime, where there is a limited supply of lubricant to the contact due to greases being squeezed out of the contact zones under small-amplitude oscillations or vibrations $[4,6,10,11]$. Consequently, the development of ZDDP tribofilm on the worn surface can be slow and uneven. Noticeably, with the less intensive parameters applied in the current work (i.e., low frequency, normal pressure, sliding velocity), thus the formation of tribofilm seems less evident than those reported in the literature. With respect to the false brinelling test rig experiments, an increase in the local temperature due to a higher frequency $(24 \mathrm{~Hz})$, higher pressure and longer testing durations seems reasonable, suggesting the relative thick and evenly distributed tribofilm can certainly form on the contacting surfaces, therefore, no distinguishing wear marks were found in the case of PU/ $\mathrm{PFPE}+\mathrm{E}$ and $\mathrm{LiC} / \mathrm{Syn}+\mathrm{M}$.

\subsection{Tribological Properties of ZDDP-Containing Greases}

The reciprocating wear tests indicated $\mathrm{LiC} / \mathrm{Syn}+\mathrm{M}$ imparted better wear resistance than PU/PFPE $+\mathrm{E}$ due to the lowest measured COF $(<0.05)$ under the studied conditions. The difference in COF measurements can be attributed to thickener types and the polarity of base oils present in the greases, leading to different tribological performance. As reported by Suarez et al. [22, 47], polar base oil (i.e., PFPE + E) impinges on ZDDP molecules accessing the contact surface of the steel, leading to lower adsorption rates than ZDDP blended in a non-polar (or less polar oil, i.e., Syn + M). This results in a thinner layer of tribofilm growth and influencing tribological performance. Suarez et al. [22, 47] also found that the ZDDP-derived tribofilm grew slowly when blending in the polar base oil than nonpolar base oil. Furthermore, the present types of thickeners in the greases adds the complexity of ZDDP-induced tribofilm formation on the steel surfaces due to the competitive interactions between ZDDP-thickener and the thickener-steel surface. For example, it was reported that adding ZDDP either in Li grease or polypropylene grease resulted 
in better performance than in $\mathrm{LiC}$ grease under the tested conditions $[46,48,49]$. Considering that the volume of the base oil is generally up to $70 \%$ and given that the remainder is thickener and less than 5\% additives, the polarity of base oil would have stronger impact on the formation of ZDDP tribofilm, therefore, influencing the variation in tribological performance (shown in COF measurements) of the two greases as seen in reciprocating wear tests.

\subsection{False Brinelling Wear in Li/M and Li/Syn}

Compared to PU/PFPE + E and LiC/Syn $+\mathrm{M}$, shallower wear depths were found under $\mathrm{Li} / \mathrm{Syn}$ and $\mathrm{Li} / \mathrm{M}$ lubricated conditions after false brinelling tests. The performance of false brinelling reduction observed in $\mathrm{Li} / \mathrm{Syn}$ and $\mathrm{Li} / \mathrm{M}$ is associated with the consistency of the grease, thickener structure as well as the polarity of the base oil. From Fig. 3, $60 \%$ wear depth reduction was found in $\mathrm{Li} / \mathrm{Syn}$ compared to $\mathrm{Li} / \mathrm{M}$. One explanation is the structure of $\mathrm{Li} / \mathrm{Syn}$ is less rigid and the thickener loosely connected (NLGI $=1$ ) compared to Li/M (NLGI =3). As shown in Fig. 9c, the degradation of thickener in Li/Syn was observed after low frequency reciprocating wear tests at $8 \mathrm{~Hz}, 340 \mathrm{MPa}$. Hence, the structural integrity of Li/Syn thickener degrades easily, leading to potential accumulation of oil and potential thickener into the fretting contact areas [17]. The oil bleed seems to be one of the contributing factors, as more oil was bled out from $\mathrm{Li} / \mathrm{Syn}(0.5 \%)$ compared to $\mathrm{Li} / \mathrm{M}(0.15 \%)$ in the first $5 \mathrm{~h}$ (Fig. 10). It is known that the polar base oil molecules has higher affinity to adhere to the steel surface compared to non-polar oil. Therefore, the ability of synthetic oil in Li/ Syn reacts with the steel metal to form the lubrication film is considerably easier than mineral oil in $\mathrm{Li} / \mathrm{M}$. The above results suggested that $\mathrm{Li} / \mathrm{Syn}$ has a more abundant and less viscous oil, which bleeds readily from the more opening structured thickener compared to $\mathrm{Li} / \mathrm{M}$. These variables would have contributed synergistically to reduce the false brinelling wear depth.

It is noted that, for $\mathrm{Li} / \mathrm{M}$ and $\mathrm{Li} / \mathrm{Syn}$, the mechanism preventing the fretting wear are different to Maruyama et al. [17], where high oil separation and viscosity does not contribute to fretting wear reduction. Instead, it was a layer of residual thickener found in the fretting contact that effectively prevented fretting wear. Nogi et al. [39] theoretically indicated that the concentration of thickener increases surrounding EHD contact. The different findings between above research and the current work could be due to the types of thickeners (thickener geometry) and base oil (polarity and viscosity); polyurea as used in the study by Maruyama et al. [17] is generally platelets/spherical shapes [30], whereas the lithium thickener in the present work is fibrous. In Nogi et al. work [39], grease with high polarity and low viscosity was used to predict the grease film thickness. As suggested by
Cyriac et al. [30] and Saatchi [14], the geometry of thickener and the ratio of thickener volume fraction to oil, impact on how thickener transport, structural squeezing and thickener rotation through the tribological contact areas occurs.

\subsection{Greases with ZDDP Versus EP}

From Fig. 4b, the result indicated ZDDP-formed tribofilm offers superiors friction and wear resistance than EP-formed sulfide layer as evidenced by lower COF values of PU/ $\mathrm{PFPE}+\mathrm{E}$ and $\mathrm{LiC} / \mathrm{Syn}+\mathrm{M}$. Furthermore, no false brinelling wear was observed in the raceways for ZDDP-containing greases but EP-containing grease $(\mathrm{Li} / \mathrm{M})$. One explanation can be the ZDDP-formed film is more resistant to wear than EP-formed film, which was demonstrated by Hao et al. through the scratching tests on the thermal-induced films (i.e., $600{ }^{\circ} \mathrm{C}$ ) by both ZDDP and EP on high-speed steel material [50]. In this study, a more brittle oxide iron layer $\left(\mathrm{Fe}_{2} \mathrm{O}_{3}\right)$ was found on the EP-induced film, which was absent in ZDDP-induced film under the studied conditions. Future study could include a greater variety of grease formula, such as a grease with same base oil and same type of thickener, but pairing with different additives (i.e., ZDDP versus EP), so the effect of additives on false brinelling wear mitigation under grease lubricated conditions can be clearer.

\subsection{Oil Bleed}

In Sect. 2.6, the 'dynamic bleed' was introduced based on the assumption that, in the tribological contact, oil release mechanism under small oscillations or vibrations (during transportation) which would be different from the standard bleed rate test, refers as 'static bleed'. Interestingly, PU/ PFPE + E has the worst 'dynamic oil bleed' compared to the other greases (Fig. 10), whereas the 'static oil bleed' data indicated Li/M has the worse oil bleed (oil separation $=0.6$ in Table 1). The difference observed in' static' and 'dynamic' oil bleed would be due to the geometry of the thickener types as well as the consistency of the greases (amount of base oil). There is less PU thickener (indicated by $\mathrm{NLGI}=2$, lower consistency) than Li thickener in the grease (NLGI $=3$, higher consistency). That means that the degree of dilution for PU thickener is greater in PU/ $\mathrm{PFPE}+\mathrm{E}$ than the $\mathrm{Li}$ thickener in $\mathrm{Li} / \mathrm{M}$ and yet the plate/ particle-like PU and short fibres structure (Fig. 9c) do not impede the oil released from the grease in the 'static bleed' test. In 'dynamic bleed', viscosity of the oil and the geometry of thickener are contributing factors to the lower oil bleed found in PU/PFPF + E (Fig. 10). That said, PU/ $\mathrm{PFPE}+\mathrm{E}$ contains a base oil with higher viscosity and the plate/particle-like PU structure could readily move forming a more closely packed structure under centrifugal force, hence, affecting the flow of oil in the effective thickener structure. It 
is interesting to discover that although PU/PFPE $+\mathrm{E}$ shows the slowest oil bleed in the dynamic condition (Fig. 10), the grease can reduce the false brinelling wear as visualised on the raceway (Fig. 3b). This finding also highlights the effectiveness of ZDDP on false brinelling wear resistance.

Our results show that ZDDP, as one of most commonly used antiwar agents, mitigates false brinelling of roller bearings occurring rail/sea transportation. To broaden the scope of this research, future work can include grease with other AW agents, which will help identify the optimal grease formula to prevent false brinelling of roller bearings. Finally, it is noted, that from this study there are multiple variables that affect the formation of a tribofilm in cases of false brinelling. Whilst this study confirms that the presence of a tribofilm as a major contributor to the mitigation of false brinelling damage, an in-depth investigation into the tribofilm formation mechanisms as well as the thickness of the tribofilm, were beyond the scope of this study. Hence, future investigations on tribofilm formation under formation under false brinelling conditions is warranted.

\section{Conclusion}

False brinelling test rig experiments were conducted to simulate damage occurring in grease lubricated roller bearings during rail/sea transportation. The effect of four types of greases with different thickeners and base oils on false brinelling damage were assessed. The results of the current study demonstrates that the magnitude of false brinelling wear reduction under grease lubricated conditions various significantly, influenced by the grease formula, as summarized below:

- ZDDP-containing greases had superior performance mitigating false brinelling as evidenced by no wear marks was observed on the inner raceway. The predominant mechanism of false brinelling reduction was due to the formation of a ZDDP tribofilm on the contact.

- The results highlight that, for grease lubricated contacts, ZDDP tribofilms can form by heat generated in the presence micro-sliding motions and energy dissipation at the interface at low frequencies $(4-8 \mathrm{~Hz})$ acting in conjunction with normal pressure, due to the mechanochemical reactions.

- Two conditions facilitated the formation of ZDDPderived tribofilm under grease lubricated conditions: either (1) $8 \mathrm{~Hz}, 340 \mathrm{MPa}, 25{ }^{\circ} \mathrm{C}$ or (2) $4 \mathrm{~Hz}, 700 \mathrm{MPa}$, $25{ }^{\circ} \mathrm{C}$.

- $\mathrm{COF}$ of $\mathrm{LiC} / \mathrm{Syn}+\mathrm{M}$ is $50 \%$ lower than that of PU/ $\mathrm{FPFE}+\mathrm{E}$ under the studied conditions. The difference in tribological properties observed in both ZDDP-containing greases can be attributed to the difference in polarity of the base oil and the types of thickeners used in the greases.

- Grease with EP (Li/M) and Li/Syn can reduce false brinelling wear by $~ 92 \%$ and $97 \%$ compared to the dry condition, respectively. The mechanism of false brinelling reduction observed in $\mathrm{Li} / \mathrm{Syn}$ and $\mathrm{Li} / \mathrm{M}$ is associated by the synergistic effect of: consistency of the grease, thickener structure as well as the polarity of the base oil. Compared to Li/M, Li/Syn has a more abundant and less viscous oil, which bleeds readily from the more open structured thickener. Furthermore, the synthetic base oil in Li/Syn has higher affinity to adhere to the steel surface compared to mineral oil in $\mathrm{Li} / \mathrm{M}$.

The current study highlighted greases with ZDDP (PU/ $\mathrm{PFPE}+\mathrm{E}$ and $\mathrm{LiC} / \mathrm{Syn}+\mathrm{M}$ ) provide the best resistance for false brinelling damage. The results also indicate that the magnitude of false brinelling wear reduction under grease lubricated conditions is significantly influenced by the grease formula. Future research into false brinelling mitigation and prevention could include an in-depth investigation into the mechanisms and variables (e.g., additive types, wide temperature/vibration ranges) that affect the tribofilm formation.

Acknowledgements The authors greatly appreciate the financial support from the Rail Manufacturing Cooperative Research Centre (funded jointly by participating rail organisations and the Australian Federal Government's Business Cooperative Research Centres Program) through Project "Monitoring and Control of False Brinelling".

Funding Open Access funding enabled and organized by CAUL and its Member Institutions.

Open Access This article is licensed under a Creative Commons Attribution 4.0 International License, which permits use, sharing, adaptation, distribution and reproduction in any medium or format, as long as you give appropriate credit to the original author(s) and the source, provide a link to the Creative Commons licence, and indicate if changes were made. The images or other third party material in this article are included in the article's Creative Commons licence, unless indicated otherwise in a credit line to the material. If material is not included in the article's Creative Commons licence and your intended use is not permitted by statutory regulation or exceeds the permitted use, you will need to obtain permission directly from the copyright holder. To view a copy of this licence, visit http://creativecommons.org/licenses/by/4.0/.

\section{References}

1. Massi, F., Rocchi, J., Culla, A., Berthier, Y.: Coupling system dynamics and contact behaviour: modelling bearings subjected to environmental induced vibrations and 'false brinelling' degradation. Mech. Syst. Signal Process. 24, 1068-1080 (2010)

2. Silva, J., Cardoso, A.J.M.: Bearing failures diagnosis in threephase induction motors by extended Park's Vector approach. In: Proceedings of the 31st Annual Conference of IEEE Industrial Electronics Society, p. 6 (2005) 
3. Yano, A., Noda, Y., Akiyama, Y., Watanabe, N., Fujitsuka, T.: Evaluation of fretting protection property of lubricating grease applied to thrust ball bearing-Comparison between ASTM D4170 test and impact fretting test. Tribology 5, 52-59 (2010)

4. Greco, A., Sheng, S., Keller, J., Erdemir, A.: Material wear and fatigue in wind turbine systems. Wear 302, 1583-1591 (2013)

5. Fallahnezhad, K., Liu, S., Brinji, O., Marker, M., Meehan, P.A.: Monitoring and modelling of false brinelling for railway bearings. Wear 424-425, 151-164 (2019)

6. Zhou, Z.R., Vincent, L.: Lubrication in fretting: a review. Wear 225-229, 962-967 (1999)

7. Godfrey, D.: Fretting corrosion or false brinelling? Tribol. Lubr. Technol. 59, 28-30 (2003)

8. Kontou, A., Taylor, R.I., Spikes, H.A.: Effects of dispersant and ZDDP additives on fretting wear. Tribol. Lett. 69, 6 (2020)

9. Schwack, F., Prigge, F., Poll, G.: Finite element simulation and experimental analysis of false brinelling and fretting corrosion. Tribol. Int. 126, 352-362 (2018)

10. Errichello, R.: Another perspective: false brinelling and fretting corrosion. Lubr. Eng. 60, 34-36 (2004)

11. Schwack, F., Bader, N., Leckner, J., Demaille, C., Poll, G.: A study of grease lubricants under wind turbine pitch bearing conditions. Wear 454-455, 203335 (2020)

12. Fallahnezhad, K., Brinji, O., Desai, A., Meehan, P.A.: The influence of different types of loading on false brinelling. Wear 440, 203097 (2019)

13. Zhou, Z.R., Kapsa, P., Vincent, L.: Grease lubrication in fretting. J. Tribol. 120, 737-743 (1998)

14. Saatchi, A.: The effect of grease composition on fretting wear. University of Akron (2019)

15. Shima, M., Suetake, H., McColl, I.R., Waterhouse, R.B., Takeuchi, M.: On the behaviour of an oil lubricated fretting contact. Wear 210, 304-310 (1997)

16. Maruyama, T., Saitoh, T.: Oil film behavior under minute vibrating conditions in EHL point contacts. Tribol. Int. 43, 1279-1286 (2010)

17. Maruyama, T., Saitoh, T., Yokouchi, A.: Differences in mechanisms for fretting wear reduction between oil and grease lubrication. Tribol. Trans. 60, 497-505 (2017)

18. Liu, Q.Y., Zhou, Z.R.: Effect of displacement amplitude in oillubricated fretting. Wear 239, 237-243 (2000)

19. Pittroff, H.: Fretting corrosion caused by vibration with rolling bearings stationary. J. Basic Eng. 87, 713-723 (1965)

20. Kita, T., Yamamoto, Y.: Fretting wear performance of lithium 12-hydroxystearate greases for thrust ball bearing in reciprocating motion. JPN J. Tribol. 42, 492-499 (1997)

21. Tomala, A., Naveira-Suarez, A., Gebeshuber, I.C., Pasaribu, R.: Effect of base oil polarity on micro and nanofriction behaviour of base oil + ZDDP solutions. Tribol. Mater. Surf. Interfaces 3, 182-188 (2009)

22. Naveira Suarez, A., Grahn, M., Pasaribu, R., Larsson, R.: The influence of base oil polarity on the tribological performance of zinc dialkyl dithiophospate additives. Tribol. Int. 43, 2268-2278 (2010)

23. Grahn, M., Naveira-Suarez, A., Pasaribu, R.: Effect of ZDDP on friction in fretting contacts. Wear 273, 70-74 (2011)

24. Topolovec-Miklozic, K., Forbus, T.R., Spikes, H.A.: Film thickness and roughness of ZDDP antiwear films. Tribol. Lett. 26, 161-171 (2007)

25. Zhang, J., Spikes, H.: On the mechanism of ZDDP antiwear film formation. Tribol. Lett. 63, 24 (2016)

26. Lin, C.-L., Meehan, P.A.: Microstructure characterization of degraded grease in axle roller bearings. Tribol. Trans. 62, 667687 (2019)

27. Lugt, P.M.: A review on grease lubrication in rolling bearings. Tribol. Trans. 52, 470-480 (2009)
28. De Laurentis, N., Kadiric, A., Lugt, P., Cann, P.: The influence of bearing grease composition on friction in rolling/sliding concentrated contacts. Tribol. Int. 94, 624-632 (2016)

29. Gonçalves, D., Graça, B., Campos, A.V., Seabra, J., Leckner, J., Westbroek, R.: Formulation, rheology and thermal ageing of polymer greases-Part I: influence of the thickener content. Tribol. Int. 87, 160-170 (2015)

30. Cyriac, F., Lugt, P.M., Bosman, R., Padberg, C.J., Venner, C.H.: Effect of thickener particle geometry and concentration on the grease EHL film thickness at medium speeds. Tribol. Lett. 61, 18 (2016)

31. Smith, G.C., Bell, J.C.: Multi-technique surface analytical studies of automotive anti-wear films. Appl. Surf. Sci. 144-145, 222-227 (1999)

32. Heuberger, R., Rossi, A., Spencer, N.D.: XPS study of the influence of temperature on ZnDTP tribofilm composition. Tribol. Lett. 25, 185-196 (2007)

33. Nedelcu, I., Piras, E., Rossi, A., Pasaribu, H.R.: XPS analysis on the influence of water on the evolution of zinc dialkyldithiophosphate-derived reaction layer in lubricated rolling contacts. Surf. Interface Anal. 44, 1219-1224 (2012)

34. Martin, J.M.: Antiwear mechanisms of zinc dithiophosphate: a chemical hardness approach. Tribol. Lett. 6, 1-8 (1999)

35. Kasrai, M., Fuller, M.S., Bancroft, G.M., Yamaguchi, E.S., Ryason, P.R.: X-Ray absorption study of the effect of calcium sulfonate on antiwear film formation generated from neutral and basic ZDDPs: Part 2-sulfur species. Tribol. Trans. 46, 543-549 (2003)

36. Martin, J.M., Grossiord, C., Varlot, K., Vacher, B., Igarashi, J.: Synergistic effects in binary systems of lubricant additives: a chemical hardness approach. Tribol. Lett. 8, 193-201 (2000)

37. Soltanahmadi, S., Esfahani, E.A., Nedelcu, I., Morina, A., van Eijk, M.C.P., Neville, A.: Surface reaction films from amine-based organic friction modifiers and their influence on surface fatigue and friction. Tribol. Lett. 67, 80 (2019)

38. Cabán-Acevedo, M., Faber, M.S., Tan, Y., Hamers, R.J., Jin, S.: Synthesis and properties of semiconducting iron pyrite $\left(\mathrm{FeS}_{2}\right)$ nanowires. Nano Lett. 12, 1977-1982 (2012)

39. Nogi, T., Soma, M., Dong, D.: Numerical analysis of grease film thickness and thickener concentration in elastohydrodynamic lubrication of point contacts. Tribol. Trans. 63, 924-934 (2020)

40. Zhang, J., Ueda, M., Campen, S., Spikes, H.: Boundary friction of ZDDP tribofilms. Tribol. Lett. 69, 8 (2020)

41. Soltanahmadi, S., Morina, A., van Eijk, M.C.P., Nedelcu, I., Neville, A.: Experimental observation of zinc dialkyl dithiophosphate (ZDDP)-induced iron sulphide formation. Appl. Surf. Sci. 414, 41-51 (2017)

42. Ito, K., Martin, J.M., Minfray, C., Kato, K.: Formation mechanism of a low friction ZDDP tribofilm on iron oxide. Tribol. Trans. 50, 211-216 (2007)

43. Fujita, H., Spikes, H.A.: The formation of zinc dithiophosphate antiwear films. Proc. Inst. Mech. Eng. 218, 265-278 (2004)

44. Fujita, H., Glovnea, R.P., Spikes, H.A.: Study of zinc dialkydithiophosphate antiwear film formation and removal processes, Part I: experimental. Tribol. Trans. 48, 558-566 (2005)

45. Lugt, P.M.: Lubrication mehanisms. In: Lugt, P.M. (ed.) Grease Lubrication in Rolling Bearings, pp. 5-21. Wiley, Hoboken (2012)

46. Shu, J., Harris, K., Munavirov, B., Westbroek, R., Leckner, J., Glavatskih, S.: Tribology of polypropylene and Li-complex greases with ZDDP and MoDTC additives. Tribol. Int. 118, 189195 (2018)

47. Suarez, N.A.: The Behaviour of Antiwear Additives in Lubricated Rolling-Sliding Contacts, Machine Elements. Lulea University of Technology, Sweden (2011) 
48. Dixena, R.K., Sayanna, E., Badoni, R.P.: A study on tribological behaviours of ZDDP in polymer thickened lubricating greases. Lubr. Sci. 28, 177-186 (2016)

49. Sivik, M., Zeitz, J.B., Bayus, D.: Interactions of a zinc dithiophosphate with lithium 12-hydroxystearate grease. NLGI Spokesm. 66, 20-24 (2002)

50. Hao, L., Jiang, Z., Wei, D., Zhao, Y., Zhao, J., Luo, M., Ma, L., Luo, S., Jiang, L.: Effect of extreme pressure agents on the anti-scratch behaviour of high-speed steel material. Tribol. Int. 81, 19-28 (2015)

Publisher's Note Springer Nature remains neutral with regard to jurisdictional claims in published maps and institutional affiliations. 